senschaftler innovative Sicherheitsmechanismen an realen Beispielen umsetzen, beispielsweise in medizinischen Implantaten.

Weitere Informationen: http://www.hgi.rub.de/hgi/news/

\section{ENISA: Videos zur Sensibilisierung für Informationssicherheit}

ENISA (die EU-Agentur für Cyber-Sicherheit) stellt eine Serie von kostenlosen Videos in allen 23 EU-Amtssprachen vor. Sie haben den Zweck, das Bewusstsein für Risiken der Informationssicherheit und den sicheren Umgang mit elektronischen Daten zu stärken: 13 Videos decken eine breite Palette von Themen ab: von sicheren Passwörtern und dem Schutz sensibler Daten bis zum Sperren und Absichern des Computers. Die Videos stehen auf der Website der Agentur zum Download zur Verfügung und sind zum Einsatz im Rahmen von Seminaren über Informationssicherheit und anderen Initiativen zur Sensibilisierung frei verwendbar.

„Wir waren der Ansicht, dass es wichtig sei, die Videos in allen Amtssprachen der EU zur Verfügung zu stellen, um damit die größte Wirkung bei allen Bürgern, Unternehmen und Regierungsstellen der EU zu erzielen", sagte Professor Udo Helmbrecht, Geschäftsführender Direktor von ENISA. "Cyber-Sicherheit ist eine gemeinsame Verantwortung. In der heutigen Welt mit E-Commerce, Social Media und Kommunikation rund um die Uhr ist es von grundlegender Bedeutung, dass wir uns alle der Risiken bewusst sind und einfache Schritte beachten, um unsere Identität und Informationen zu schützen."

Diese Kampagne ist ein Teil des anhaltenden Engagements von ENISA, das Bewusstsein über Informationssicherheit zu stärken. Sie unterstreicht auch die Ziele der EU/US-Arbeitsgruppe über CyberSicherheit und Cyber-Kriminalität, deren Aufgabe darin besteht, durch Zusammenarbeit eine zuverlässige, widerstandsfähige und glaubwürdige digitale Infrastruktur zu schaffen. Das US-Heimatschutzministerium stellte die Videos von ENISA während einer von vier Themenwochen während des Nationalen Monats zur Sensibilisierung für Cyber-Sicherheit, der schon im achten Jahr in Folge organisiert wird. Der diesjährige Schwerpunkt lag auf der Online-Sicherheit für kleine und mittlere Unternehmen.

Hier können die Videos heruntergeladen werden: http://www. enisa.europa.eu/act/ar/material/awareness-raising-video-clips

\section{Samsung erschließt Nutzung von Trusted Computing-Funktionen}

Samsung engagiert sich verstärkt für eine hardwarebasierte Sicherheit in PCs, Tablets und Consumer-Geräten. wird Wave wird dafür technische Dienste, Beratungsleistungen, Validierungen sowie eine spezifisch angepasste Version seiner lokalen Management-Software für Samsungs TPM (Trusted Platform Module)-Sicherheitschips für den OEM-Vertrieb zur Verfügung stellen.

Samsung ist einAnbieter von hochentwickelten Halbleiterlösungen, die exzellentes Design, moderne Herstellungsprozesse, hohe Qualität und innovative Verpackungstechnologie vereinen. Zu seinem breiten Portfolio zählen unter anderem Sicherheitschips für Smartcards und NFC-fähige Mobilgeräte.

Wave (http://www.wave.com/) verfolgt seit Jahren den Ansatz die in die Endpunkt-Rechnerplattform selbst eingebauten Sicherheitsfunktionen wirksam zu verstärken. In diesem Segment ist Wave mit einem Sicherheitsmanagementsystem zur Datensicherung und für die Geräte-/ Nutzerauthentifizierung führend.

Durch seine Arbeit als Gründungs- und Boardmitglied der Trusted Computing Group (TCG) wirkte das Unternehmen auch maßgeblich bei der Ausgestaltung von Trusted Computing Standards mit.

\title{
Rezensionen
}

\section{Veranstaltungen}

\author{
Karl Rihaczek \\ 35. DAFTA, 17. bis 18. November 2011 in Köln \\ Die GDD Gesellschaft für Datenschutz und Datensicherung veran- \\ staltete am 17. und 18. November 2011 im Kölner Maternushaus \\ ihre „35. DAFTA Datenschutzfachtagung. Am Donnerstagvormit- \\ tag wurden, wie üblich, drei Vorträge und eine Podiumsdiskussion \\ geboten. Am Nachmittag und am Freitagvormittag liefen neben- \\ einander insgesamt 9 "Foren" und ein Spezialforum ab. Drei davon \\ wurden am Donnerstagnachmittag in einer zweiten Runde wieder- \\ holt. Am Freitagvormittag wurde der GDD Wissenschaftspreis ver- \\ liehen. Der übliche Vortrag zum erbaulichen Abschluss hieß: „Wel- \\ come to the World 2021 - eine Reise durch das Trenduniversum". \\ Die Veranstaltung stand unter dem Motto "Neues Datenschutz- \\ recht aus Brüssel und Berlin“. Aus Berlin war vom Bundesministe- \\ rium des Innern an Stelle der angesagten Staatssekretärin Corne- \\ lia Rogall-Grothe Ministerialdirektor Karl-Hans von Knobloch er- \\ schienen: Die Bundesregierung beabsichtige nicht, die virulen- \\ te Situation in den sozialen Netzwerken gesetzlich zu regeln. Sie
}

vertraue auf eine Selbstregulierung. So erwarte sie bei der Frage des Internet-„,Gefällt mir“-Buttons, dass sich eine Regelung durch Rechtsprechung ergibt.

Der zweite Redner war Paul Nemitz aus Brüssel, Direktion Grundrecht: Die EU-Kommission beabsichtige, im Januar nächsten Jahres eine Verordnung zu erlassen und wolle damit unmittelbar und zwingend das europäische Datenschutzrecht vereinheitlichen. Es solle nur eine zentrale europäische Aufsichtsbehörde geben. An Stelle des derzeitigen Meldesystems sollen betriebliche Datenschutz-beauftragte europaweit bestellt werden müssen. Über die Bestellungsvorschriften und eine mit ihnen kleineren deutschen Unternehmen gegebene Möglichkeit, betriebliche Datenschutzbeauftragte abzubestellen, konnte Herr Nemitz noch nichts sagen. Im Übrigen war an diesem Vormittag beherrschendes Thema der Beschäftigten-Datenschutz. Dazu trug Gregor Thüsing, Universität Bonn, vor. Er kritisierte den Entwurf des Bundesministeriums des Innern als handwerklich schlecht gemacht, unklar und mit Redundanzen, die die Spuren konfligierender Regelungsabsichten erkennen lassen. 